\title{
Analytical modelling of Kirschner wires in Ilizarov circular external fixators using a tensile model
}

\author{
A R Zamani and S O Oyadiji* \\ School of Mechanical, Aerospace and Civil Engineering, University of Manchester, Manchester, UK
}

The manuscript was received on 8 November 2007 and was accepted after revision for publication on 4 March 2008.

DOI: 10.1243/09544119JEIM373

\begin{abstract}
Mechanical characteristics of orthopaedic fixators, such as the stiffness and stability, directly influence the mechanobiological environment in which the bone is healed. In circular external fixators, the transfixing Kirschner wires are the major contributors to the biomechanics involved. A comprehensive understanding of the mechanical behaviour of the wires is therefore the key to biomechanical analysis of the Ilizarov fixator. In this study, to model the behaviour of the wires, a purely theoretical approach has been adopted to obtain explicit equations for a solely tension-based model formulation. Mathematical modelling leads to new algebraic polynomials whose solutions are the exact maximum deflection, angle of deflection, and total (final) tension in the wire. The predictions are compared with published experimental and computational findings on the deflection and stiffness of the wires, and analytical explanations are provided for the previously observed behaviours. Parametric (practical) implications of this type of abstraction include the fact that the angle of deflection as well as the tension in the wire are independent of its length. The inverse proportionality of wire stiffness to its length is also deduced. The findings are applicable to tensile elements (ropes, chains, etc.), provided that the tensile deformation (elongation) can be deemed to be the dominant mode of deformation.
\end{abstract}

Keywords: Kirschner wires (K-wires), Ilizarov fixator, pretension of fixator wires, transverse stiffness of fixator wires, geometric non-linearity

\section{INTRODUCTION}

The Ilizarov appliance is a widely used modular external fixation system with increasing applications for stabilization of fractures, treatment of skeletal trauma and abnormalities, as well as limb lengthening [1-9]. In a simple configuration, the Ilizarov frame consists of thin wires, which are drilled through the bone, then tensioned, and clamped to a set of rings (or ring sections) which are assembled together around the limb using threaded rods and nuts as shown in Fig. 1. The wires, called Kirschner wires (K-wires), enable the fixator to balance adequate axial stiffness and overall stability with flexibility to allow limited axial interfragmental

*Corresponding author: School of Mechanical, Aerospace and Civil Engineering, University of Manchester, Pariser Building, Sackville Street, Manchester, M60 1QD, UK. email: s.o.oyadiji @manchester.ac.uk dynamization (mobility). This interfragmentary micromotion (IFM) is believed to be beneficial to bone formation and healing [7-10]. IFM is the outcome of lateral deformation in transosseous K-wires [5, 11], hence the significance of the wires to mechanobiology of the healing process. The underlying mechanical principle is the capability of a fine wire to attain a much larger lateral stiffness when subjected to a considerable axial pre-tension [3].

Although much research has been performed experimentally or by finite element analysis $[\mathbf{1}, \mathbf{2}$, 4-6, 11-14] to study the mechanical behaviour of Kwires, a purely theoretical analysis will be beneficial for a more comprehensive understanding of the problem, which will facilitate parametric studies of the influence of the various parameters on the kinematics of the fixators. Such studies will aid the current need to understand the influence of static and dynamic loads on the fracture-healing process. Thus, in this work, a purely mathematical approach 


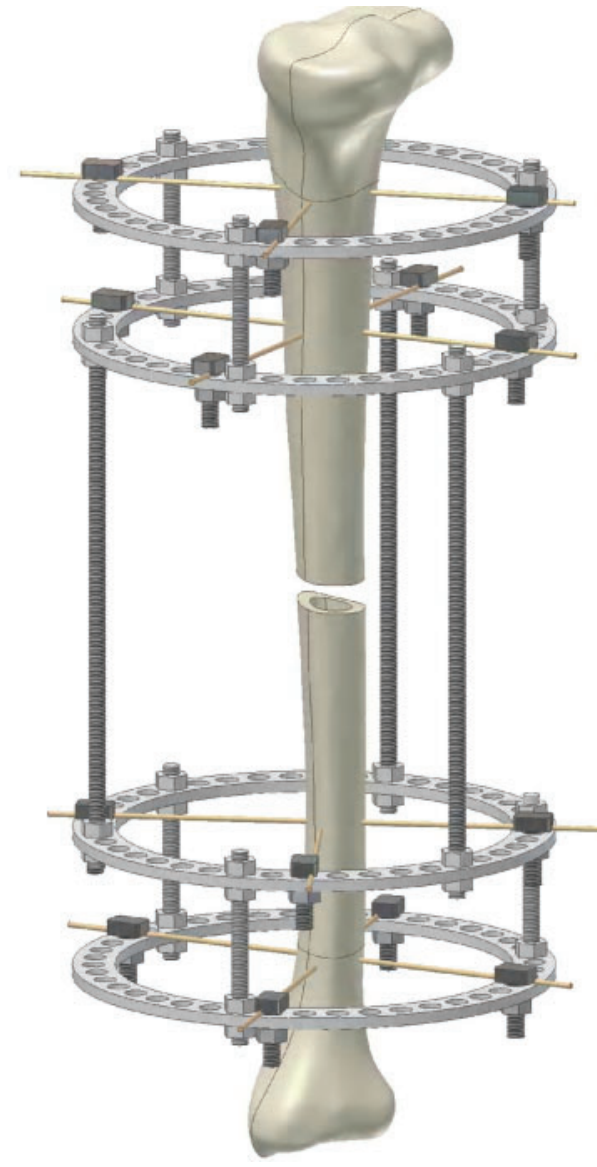

Fig. 1 Full-ring Ilizarov fixator applied for lengthening of tibia

is used to model the Kirschner wires under a lateral load.

K-wires are capable of withstanding transverse loading by being both bent and tensioned (including pre-tension), and it is the magnitude of the applied loads (pre-tension and transverse load) that determines which of these two modes of deformation is dominant. Two essentially different models have been adopted to describe theoretically the mechanical behaviour of the tensioned K-wires under a transverse load:

(a) a beam under simultaneous axial tension and transverse loading [6];

(b) pre-tensioned wire (or chain) subjected to a lateral load $[\mathbf{4}, \mathbf{5}]$.

The bases for these two models are mutually exclusive. The first model generally regards the bending moment as the sole cause for deformation (i.e. pure bending) and totally ignores any tensile effect, such as elongation, in the beam. Therefore, it can be called a flexural model and takes the bending moment due to the action of the axial forces (here, pre-tension $F$ ) into the moment equation. However, the second model, called a tensile model, in which bending moment is totally neglected and deformation is wholly attributed to the effect of the tensile force present throughout the wire (i.e. pure tension).

It should be noted that, regarding the axially tensioned beam (ATB) model, used in reference [6], simultaneous axial tension and transverse loading mean that one load cannot affect the other. Therefore the axial tension cannot increase because of the application of the transverse load, which is the case because of the geometry of the K-wires (in terms of the high slenderness ratio $L / r_{\text {g }}$; see the Appendix for definition of the symbols). ATB solutions also predict a linear relation between transverse deflection and transverse load [15], which cannot explain the established self-stiffening effect [4] and load-deflection non-linearity $[\mathbf{1}, \mathbf{4}, \mathbf{9}]$.

To compare their experimental results with an analytical solution, Donga et al. [6] assumed this type of modelling and used the solution from the handbook by Roark and Young [15] for maximum deflection of a beam under simultaneous axial tension and transverse loading. The handbook includes a table which gives the maximum deflections for a range of axial tensions, relative to those of the same untensioned (tension-free) beam, under the same transverse loading.

The applied transverse load $P$ should also be kept within a certain limit for the tensioned beam formulation to be applicable, which is in no way guaranteed, given the situation which the K-wires are normally subjected to. There are also other caveats involving the axial tension (here, $F$ ) and other parameters vis-à-vis the application of tensioned beam solutions to K-wires [15].

Plastic (permanent) deformation due to yielding at the clamps, as well as the subsequent release (reduction) of tension in the wires, are a major factor in determining mechanical behaviour of the K-wires in an Ilizarov device $[\mathbf{1}, \mathbf{5}, \mathbf{1 2}, \mathbf{1 4}]$. Nevertheless, here the focus is on purely linear elastic material behaviour, ignoring material non-linearity due to hardening and yielding, or non-linear elasticity.

Investigations have shown that transverse loads exceeding $50 \mathrm{~N}$ cause plastic deformation; furthermore, yielding of the material can occur because of clamping $[\mathbf{1}, \mathbf{1 2}]$. These plastic deformations undermine the flexural (ATB) model and make the case for the tensile (wire) model [5] most compelling.

The assumption of a purely tensile model is backed by published experimental [1], finite element 
$[1,12]$, and theoretical studies $[5,12]$ and also by the fact that, according to the beam-under-axial-tension formulation, the bending moment is concentrated around the point of application of the load as well as at the clamps, and rapidly diminishing elsewhere. Furthermore, the purely elastic analysis is supported by the fact that material non-linearities induced by clamping, yielding under the stress induced by bending moment and twisting of the fixation bolts, are concentrated at and around the immediate vicinity of the clamps, leaving the stress level in the wire segment covering the bone-clamp distance within the elastic range. For the reasons given above, it can be imagined that the part of the wire under or in the vicinity of the clamps yields and starts to deform plastically, while the wire segment between the bone and the clamps is still deforming elastically.

Therefore, at relatively large lateral loads, which are representative of the typical lateral loads to which the K-wires are subjected in most applications, they need to be treated as wires. Here, as in many other cases, the application of larger loads and its ensuing large deformations mean that geometric non-linearities should be taken into account [11]. This non-linearity of the transverse load-deflection relation and stiffness in K-wires is a well-established fact throughout the literature $[\mathbf{1}-\mathbf{4}, \mathbf{1 1}, \mathbf{1 2}]$. Thus a purely elastic analysis of the problem is justifiable.

The central loading of a beam, wire, or cable is a defining case regarding its stiffness, which means the minimum stiffness. In the case of K-wires in an Ilizarov device, it can be imagined that the minimum stiffness is what in reality occurs (e.g. if free sliding is assumed). Also, most of the experimental, finite element, or theoretical results in the literature are for the case of a centrally loaded, tensioned K-wire. Therefore it is reasonable to begin the analytical formulation with a central loading condition. Since, in the part of the wire which is trapped under the bone, there is no bending, elongation is very small, and orientation is preserved during the deformation; it can be ignored in the formulations to follow.

\section{MATHEMATICAL MODELLING OF A PRE- TENSIONED WIRE SUBJECTED TO A LATERAL LOAD}

\subsection{Review and assumptions}

A wire is considered as only being capable of withstanding a tensile force and incapable of resisting any bending. Since bending is ignored, then there can be no curvature in the deformed shape. Mathematically speaking, $y^{\prime \prime}=0$; therefore $y^{\prime}$ must be a constant. Thus the deformed shape must consist of only straight-line segments. In this formulation, deformation is solely attributed to the effect of tensile force along the wire. Hence, it can also be called the tensile model.

Sometimes this type of behaviour is referred to as purely elastic or perfectly flexible [15]. The problem with these descriptions is that they imply that this type of behaviour can be attributed to the material property of the element, while the fact is that this is more a result of geometry (e.g. slenderness ratio $L / r_{\mathrm{g}}$ ) rather than a material property.

By basing their mathematical modelling on the formulae from Leonard's [16] 1988 book, Aronson and Harp [3] in effect used the wire model to estimate deflection and stiffness of the K-wires mathematically. Nikonovas and Harrison [5] introduced a chain model, in which they assumed that the tension is uniform throughout the K-wires, regardless of the geometry of the problem (e.g. application of an off-centred load). They provided an implicit load-deflection equation which can be used to produce graphs and tables for load-deflection and stiffness where deflection can be considered as the independent variable. However, in real clinical applications, where the conditions are given in terms of pretension and applied transverse load, there was still a need for an explicit equation in terms of deflection, which can be used to calculate the deflection directly for a given set of parameters (namely $E, A, F, P$, and $L$ ). In this section, for the case of a centrally loaded wire, algebraic polynomials are derived whose solutions are maximum deflection, final tension, and angle of deflection in the wire.

Even in the absence of pre-tension $(F=0)$, this problem needs more clarification. The equation for the maximum wire deflection (i.e. equation (13) below) provides the exact solution to a classical problem of solid mechanics. Although this is the classic case of geometric non-linearity, the magnitude of which is most prominent in large deformations, it has been solved only approximately. This could mean that the solution has limitations for large deflections. In some cases, an approximate solution using binomial expansion has led to significant errors [17].

A variety of the values of Young's modulus of K-wires were found in the literature, including $E=151 \mathrm{GPa}$ [12], $E=193 \mathrm{GPa}[\mathbf{1 1}]$, and $E=197 \mathrm{GPa}$ [1]. However, the classic value of $E=200 \mathrm{GPa}$ for stainless steel is used through the rest of this paper. 


\subsection{Derivation of equations}

The definitions of the notation used are stated at the end of the paper in the Appendix. Under a given lateral load, the vertical component of $T$ does not change, and the pre-tensioning only contributes to the horizontal component of $T$ (namely $H$ ). If $\delta_{0}$ is the wire elongation due to pre-tension, $\Delta L$ is the elongation due to the sole effect of lateral loading, and $e$ is the total elongation due to pre-tension and applied lateral load, then

$$
\delta_{0}=L_{0}-L_{-1}
$$

$$
\Delta L=L^{\prime}-L_{0}
$$

$$
e=L^{\prime}-L_{-1}
$$

where $L_{-1}, L_{0}$, and $L^{\prime}$ are half the effective wire lengths (i.e. the wire segment connecting the bone to the clamp) before pre-tension, after pre-tension, and after application of pre-tension and the lateral load respectively.

Force equilibrium in Fig. 2(a) gives

$$
\sin \alpha=\frac{P}{2 T}
$$

$$
H=\sqrt{T^{2}-\left(\frac{P}{2}\right)^{2}}
$$

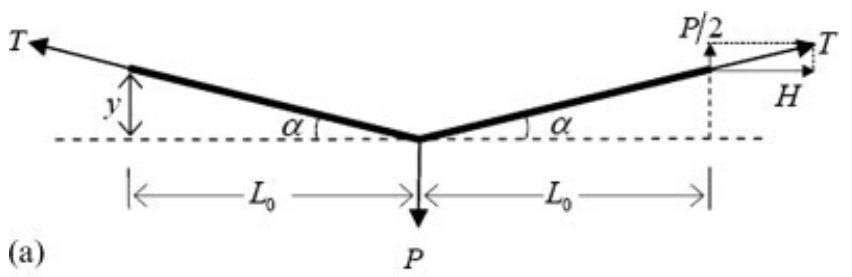

(a)

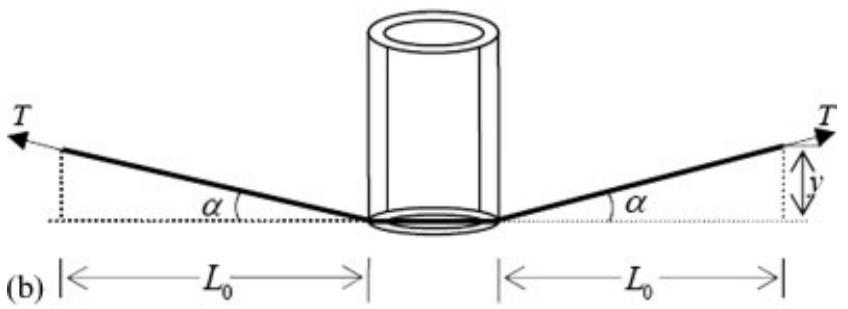

Fig. 2 (a) Wire is pre-tensioned by the axial force $F$ and fixed at both ends, subjected to a lateral load $P$; (b) wire model applied to a K-wire under the bone
It is seen from the geometry in Fig. 2(a) that the angle and length of the wire after deformation are given by

$$
\begin{aligned}
& \cos \alpha=\frac{L_{0}}{L^{\prime}} \\
& \sin \alpha=\frac{y}{L^{\prime}} \\
& L^{\prime}=\sqrt{L_{0}^{2}+y^{2}}
\end{aligned}
$$

Assuming linear elastic material behaviour means that

$$
\begin{aligned}
& \delta_{0}=\frac{F L_{-1}}{E A} \\
& e=\frac{T L_{-1}}{E A}
\end{aligned}
$$

where $F$ is the pre-tension and $T$ is the final tension in the wire after the lateral load is applied.

Substituting equation (4a) into equation (1a), and equation (4b) into equation (1c), give respectively

$$
\begin{aligned}
\frac{L_{0}}{L_{-1}} & =\frac{E A+F}{E A} \\
\frac{L^{\prime}}{L_{-1}} & =\frac{E A+T}{E A}
\end{aligned}
$$

Combining equations (3a), (5a), and (5b) gives

$$
\cos \alpha=\frac{E A+F}{E A+T}
$$

Combining equations (2a) and (6) gives

$$
\left(\frac{E A+F}{E A+T}\right)^{2}+\left(\frac{P}{2 T}\right)^{2}=1
$$

Equation (7) can be rearranged in the form

$$
\begin{aligned}
& 4 T^{4}+8 E A T^{3}-\left(P^{2}+8 E A F+4 F^{2}\right) T^{2} \\
& -2 E A P^{2} T-P^{2} E^{2} A^{2}=0
\end{aligned}
$$

Substituting $T$ from equation (4) into equation (2) and using the result in equation (3) for $\sin \alpha$ gives

$$
\frac{y}{L^{\prime}}=\frac{P}{2\left(E A e / L_{-1}\right)}
$$


Substituting $L_{0}$ from equation (5) into equation (3) gives

$$
L^{\prime}=\sqrt{\left(\frac{E A+F}{E A} L_{-1}\right)^{2}+y^{2}}
$$

Substituting $L^{\prime}$ from equation (10) into equation (1c) and then substituting the result (for $e$ ), and substituting $L^{\prime}$ from (10) into equation (9) gives

$$
\begin{aligned}
& P L_{-1}\left[\sqrt{\left(\frac{E A+F}{E A} L_{-1}\right)^{2}+y^{2}}\right] \\
& =2 E A y\left[\sqrt{\left(\frac{E A+F}{E A} L_{-1}\right)^{2}+y^{2}}-L_{-1}\right]
\end{aligned}
$$

Rearranging equation (11) into an algebraic form with respect to the powers of $y$ yields

$$
\begin{aligned}
& 4(E A)^{4}(y)^{4}-4(E A)^{3} P L_{-1}(y)^{3} \\
& +\left(E A L_{-1}\right)^{2}\left(4 F^{2}+8 E A F+P^{2}\right)(y)^{2} \\
& -4 E A(E A+F)^{2} P L_{-1}^{3}(y)+\left[P L_{-1}^{2}(E A+F)\right]^{2} \\
& =0
\end{aligned}
$$

In Ilizarov fixators the ring span $L_{0}$, rather than the wire length $L_{-1}$ before pre-tensioning, is known. Therefore it is useful to have equation (12) in terms of $L_{0}$. Substituting $L_{-1}$ from equation (5) gives

$$
\begin{aligned}
& 4(E A+F)^{2}(y)^{4}-4(E A+F) P L_{0}(y)^{3} \\
& +\left(4 F^{2}+8 E A F+P^{2}\right) L_{0}^{2}(y)^{2} \\
& -4(E A+F) P L_{0}^{3}(y)+\left(P L_{0}^{2}\right)^{2}=0
\end{aligned}
$$

To complete the set of equations with an equation directly giving the deflection angle $\alpha$, substitution of $T$ from equation (2) into equation (8) gives

$$
\begin{aligned}
& 4(E A)^{2} \sin ^{4} \alpha+4 E A P \sin ^{3} \alpha \\
& +\left(P^{2}+8 E A F+4 F^{2}\right) \sin ^{2} \alpha \\
& -4 E A P \sin \alpha-P^{2}=0
\end{aligned}
$$

In applying this model to $\mathrm{K}$-wires in an Ilizarov appliance, $L_{0}$ is the bone-clamp distance or $L_{0}=$ $D / 2-r$ (see Fig. 2(b)), where $D$ is the internal diameter of the ring and $r$ is the bone radius at the point of insertion of the wire.

\section{DISCUSSION AND IMPLICATIONS OF THE EQUATIONS}

Equations (8), (12), (13), and (14) formulate the tensile or wire model behaviour explicitly. The following results can be inferred from the equations.

\subsection{Nature of the load-deflection relation}

Equation (13) not only demonstrates the nonlinearity of the load-deflection $(P-y)$ relation but also describes the nature of this relation in terms of a mathematical equation. Although the load-deflection relation is non-linear, it is still expressed by a polynomial equation of fourth degree. Mathematically speaking, equations (8), (12), (13), and (14) can be solved exactly by algebraic methods using radicals; thus the solution can be obtained with minimal computation. Of course, there is only one acceptable solution among the possible quartet of solutions. However, experience of solving it showed that to distinguish the roots is not difficult; all negative, complex, and abnormally large or small roots can easily be ruled out.

\subsection{Geometric non-linearity}

Since linear elasticity is maintained in this formulation, the observed non-linearity is obviously due to the geometric configuration of the deformations. Here, the measured deformation is the deflection of the wire in the direction of the load applied transversely to its original axis, while the actual deformation is the wire elongation due to the tension along its direction. Thus the direction in which the load-deflection is of concern (i.e. $P$ and $y$ ) is not the same as the direction in which the material behaves linearly with load, namely $F$ and $\delta_{0}$ or $T$ and $e$. This phenomenon can be quantified; from equation (1) and equation (3) the ratio of the measured deflection $y$ to the actual elongation $\Delta L$ can be given as $(\sin \alpha) /(1-\cos \alpha)$. Therefore, in fact, although the magnitude of the transverse deflection is most prominent in large deformations, the smaller the deformation, the larger this ratio becomes. For example, if the angle of deformation is $\alpha=5^{\circ}$, the transverse deflection is almost 23 times the elongation while, for $\alpha=10^{\circ}$, this ratio is halved. This clearly proves that the measured transverse deflec- 
tion can be large while the elongation is small, and that the non-linearity is intrinsic to the problem and should be paid due attention even in small deformations. Nonetheless, the level of this non-linearity is decreased with increase in the deformation. Here, ironically, assuming that the geometric shape of a straight line is maintained means that geometric non-linearity is inherent to the solution.

\subsection{Independence of wire tension from its length}

Equation (8) does not include any term for length. This proves the independence of the tension $T$ in the wire from its length. Final tension in the wire developed by application of the transverse load $P$ is plotted in Fig. 3. It can easily yield the stress in the wire, the calculation of which will show that the stress in the wire, due to the tension alone, stays below the yield stress $\left(\sigma_{\mathrm{y}}=896 \mathrm{MPa}[\mathbf{1}]\right.$ or $\sigma_{\mathrm{y}}=685 \mathrm{MPa}$ [12]) for a relatively large range of transverse loads. Mullins et al. [2] gave the failure directly in terms of a tensile force, namely $3500 \mathrm{~N}$. It confirms the applicability of a purely elastic analysis for the solely tensile (wire) model. It also suggests that it is clamping, bending, or twisting of the clamps that causes the yielding or any plastic (permanent) deformation, any of which are concentrated around the clamps, leaving the wire segments between the clamps and the bone in the elastic behaviour zone. Since there are a variety of ring diameters, it means that theoretically the tension will be the same regardless of the size of the ring, and the surgeon can rest assured that the choice of the ring size will not affect the tension in the wires after surgery.

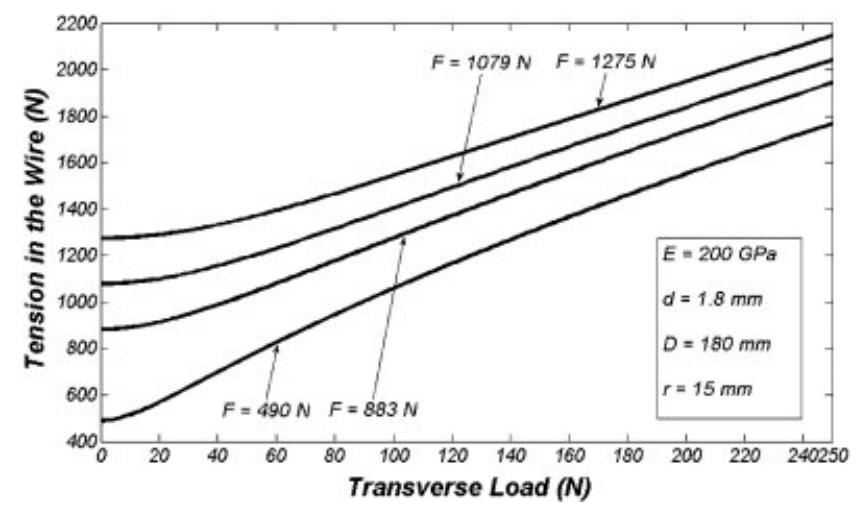

Fig. 3 Final tension $T$ in the wire as a function of applied transverse load $P$, as predicted by equation (8)

\subsection{Independence of the angle of deflection from wire length}

The length parameter is absent from equation (14), which shows the independence of the deflection angle $\alpha$ from the length of the wire or, here, the diameter of the ring. In other words, for every wire (of constant $E$ and $A$ ), under certain loading conditions $F$ and $P$, there is only one angle of deflection, regardless of the wire span (or diameter of the ring). This has implications in terms of stiffness.

\subsection{Proportionality of deflection and wire length}

Equation (13) can be considered as an implicit multivariable function, in the form of $f\left(E A, L_{0}, F, P, y\right)=0$, which suggests that $f$ is a homogeneous polynomial with respect to two variables $y$ and $L_{0}$. Therefore, if both these variables are multiplied by the same factor, the value of the expression (polynomial) is multiplied by that factor to the power of the degree of the polynomials, which, in this case, means that it stays the same (namely zero). This proves that there is a proportionality between $y$ and $L_{0}$. That is, for example, if $L_{0}$ doubles, then $y$ is also doubled. A simpler way to understand this might be to nondimensionalize the deflection $y$ by dividing equation (13) by $L_{0}^{4}$. Then it will be seen that if the ratio $y / L_{0}$ does not change, neither does the equation. This has implications in terms of stiffness, which is discussed in section 3.8 below.

\subsection{Calculation of tension loss due to slippage at the clamps}

Equations (1b), (5a), and (5b) give

$$
T=F+\frac{E A+F}{L_{0}} \Delta L
$$

Equation (15) describes the relation between the increase in the length of the wire and the increase in the wire tension due to lateral loading. It can also be used to calculate the loss in the tension owing to slippage from under the fixation bolts or ring deformation due to clamping or the bending moment. For example, in a typical case, if the wire is just to slip less than $0.25 \mathrm{~mm}$ from under each clamp (i.e. $\Delta L=0.25 \mathrm{~mm}$ ), given the parameters used above (i.e. $E=200 \mathrm{GPa}, F=1275 \mathrm{~N}, L_{0}=0.1 \mathrm{~m}$, and $\mathrm{d}=1.8 \mathrm{~mm}$ ), then the loss in tension will be $T-F=1275 \mathrm{~N}$. It can easily undo all the pre-tension in the wire. 


\subsection{Linearizing effect of pre-tension}

As the pre-tension increases, the load-deflection behaviour increasingly tends to become linear. This is shown in the shape of the load-deflection curves in Fig. 4(a); as the curves move to the right (lower pre-tensions), the curvature increases. In Fig. 5, the change in the shape of the three-dimensional surface as the pre-tension grows (almost right to left in the figure) can be considered as linearization. Therefore, the lower the pre-tension level, the higher
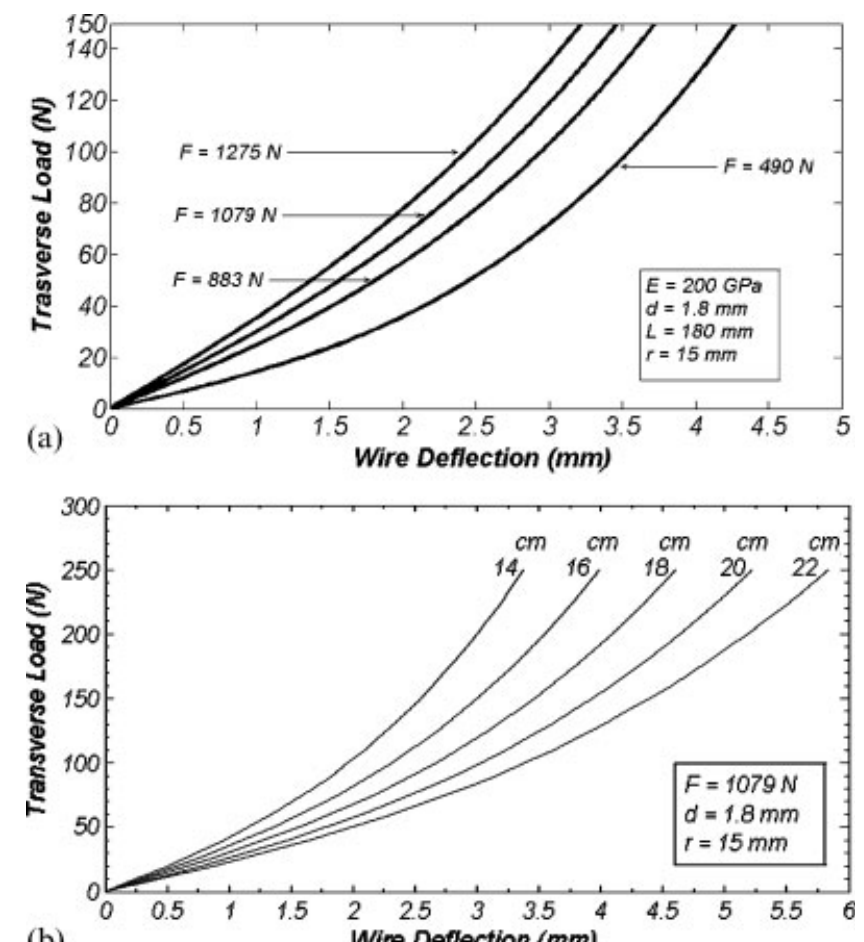

(b)

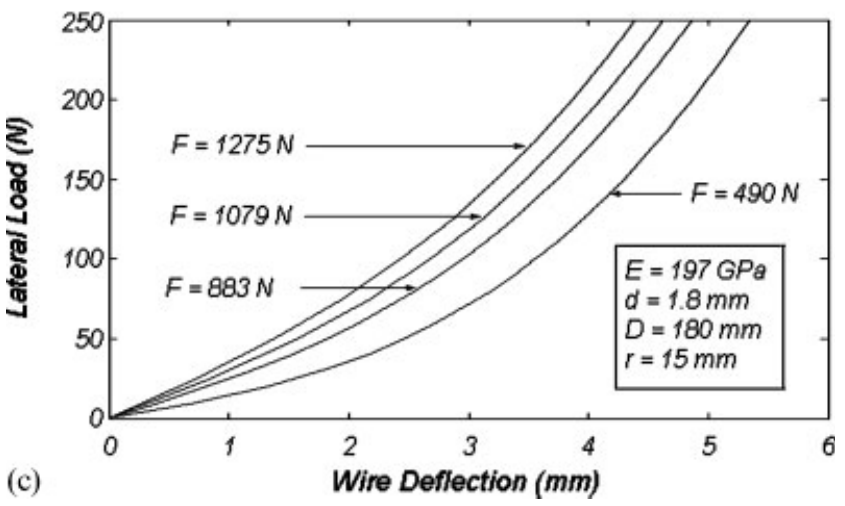

Fig. 4 Load versus deflection curves for a wire (a) under different pre-tensions where a linearizing effect of elevating the pre-tension level can be seen, (b) under a given pre-tension for a variety of wire spans, and (c) under different pre-tensions (comparable with Fig. 5 in reference [9]) is the level of load-deflection non-linearity and the ensuing self-stiffening effect. A similar effect can also be observed in Fig. 3, where elevation of the pretension level is accompanied by a less non-linear (curved) shape. When compared with experimental results (Fig. 5 in reference [9] and also Fig. 5 in reference [1]), Figs 4(a) and (c) in particular can make the effectiveness of the present elastic analysis clear to the reader.

According to the wire formulation, in the absence of pre-tension, the initial lateral stiffness will be zero at the onset of application of the vertical load, and the $P-y$ curve will be tangential to the deflection (horizontal) axis at $P=0$. Therefore, it is the pretensioning that provides the wire with the initial stiffness, which is important in the stability of fine wires in response to an impact load that occurs when the patient walks. This non-linearity may be beneficial to the mechanobiological environment at the fractured site and yield remarkable clinical results [18]. However, the pre-tensioning would mellow this non-linearity and instead provide stability, especially for shock loads (shock resistance).

\subsection{Stiffness considerations}

A stiffness discussion is crucial for stability considerations. In this section, it is established that the wire stiffness is inversely proportional to its span. From section 3.5 above, it can be said that for two different half-wire spans (or ring radii), namely $L_{01}$ and $L_{02}$, of the same type of K-wire (i.e. constant $E$ and $A$ ) under the same loading condition ( $F$ and $P$ ), the deflection angles $\alpha$ are the same for both of them. From geometry in Fig. 2(a)

$$
\tan \alpha=\frac{y}{L_{0}}=\frac{y_{1}}{L_{01}}=\frac{y_{2}}{L_{02}}
$$

The definition of stiffness as the secant modulus gives

$$
K_{\mathrm{s}}=\frac{P}{y}
$$

Equations (16) and (17) give

$$
\frac{K_{\mathrm{s} 1}}{K_{\mathrm{s} 2}}=\frac{L_{02}}{L_{01}}
$$

Stiffness can also be defined as the tangent modulus

$$
K_{\mathrm{t}}=\frac{\mathrm{d} P}{\mathrm{~d} y}=-\frac{\partial f}{\partial y} / \frac{\partial f}{\partial P}
$$




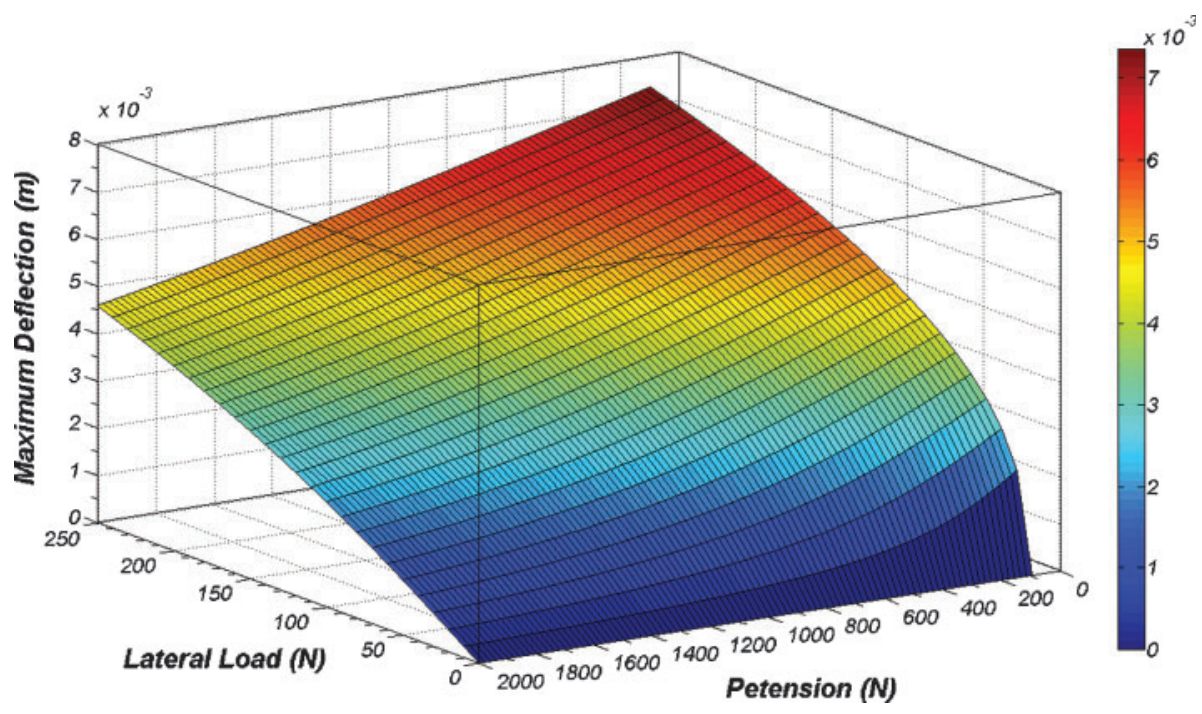

Fig. 5 A three-dimensional graph of deflection for a range of pre-tensions (100-2000 N), and a wide range of transverse loads $(0-250 \mathrm{~N})$. Here, the linearizing (flattening) effect of elevating the pre-tension level is obvious

Therefore

$$
K_{\mathrm{t}}=-\frac{16(E A+F)^{2}\left(y / L_{0}\right)^{3}-12(E A+F) P\left(y / L_{0}\right)^{2}+2\left(4 F^{2}+8 E A F+P^{2}\right)\left(y / L_{0}\right)-4(E A+F) P}{2\left[P-2(E A+F)\left(y / L_{0}\right)\right]\left[1+\left(y / L_{0}\right)^{2}\right]} \frac{1}{L_{0}}
$$

From equation (14), it is seen that $\tan \alpha$ is independent of $L_{0}$. Therefore, replacing $y / L_{0}$ in equation (20) with $\tan \alpha$ from equation (16) shows that the stiffness is inversely proportional to the half-wire length $L_{0}$. This is also confirmed by equation (18). Therefore an analogy can be made with a simple linear spring: if the length doubles, the stiffness halves. Furthermore, equation (20) demonstrates how the stiffness changes with the transverse load $P$, which is obviously nonlinear. Results from equation (20) are plotted for different wire lengths in Fig. 6(a).

Because of this inverse proportionality of lateral deflection and stiffness to the wire span, in graphs showing load-deflection or stiffness curves for different wire lengths (e.g. Figs 4(b), 6(a), and 6(b) or similar graphs), each curve (e.g. corresponding to the wire length $L_{01}$ ) can be produced by simply multiplying the abscissa (deflection or stiffness) of any of the other curves (e.g. corresponding to the wire length of $L_{02}$ ) by the proportion of their length. Therefore having one of the curves is sufficient. That is, if the curve corresponding to $L_{01}$ is $P=p_{1}(y)$ and the curve corresponding to $L_{02}$ is $P=p_{2}(y)$, then

$$
\frac{p_{2}(y)}{p_{1}(y)}=\frac{L_{01}}{L_{02}}
$$

In an experimental study on the stability in external circular fixators, based on stiffness as the measure of stability, Bronson et al. [7] found that the ring diameter affects stability (i.e. the stiffness) linearly. This corroborates the predictions of the wire formulation presented above. They also observed that a 30 per cent increase in stiffness occurs with every $40 \mathrm{~mm}$ decrease of the ring diameter. This also can be in line with the linear proportionality of the stiffness and inverse of the wire length. For example, in the tensile (wire) model, equation (21) suggests that stiffness can be formulated as

$$
K=\frac{c}{L}
$$

where $c$ is a constant independent of $L$. Testing equation (22) against the empirical finding just mentioned above gives

$$
K+0.3 K=\frac{c}{L-0.04}
$$

Equations (22) and (23) give $L \approx 170 \mathrm{~mm}$, which is near the middle of the practical range of actual ring diameters that range from 100 to $240 \mathrm{~mm}$. For this range of ring diameters, the percentage increase in 

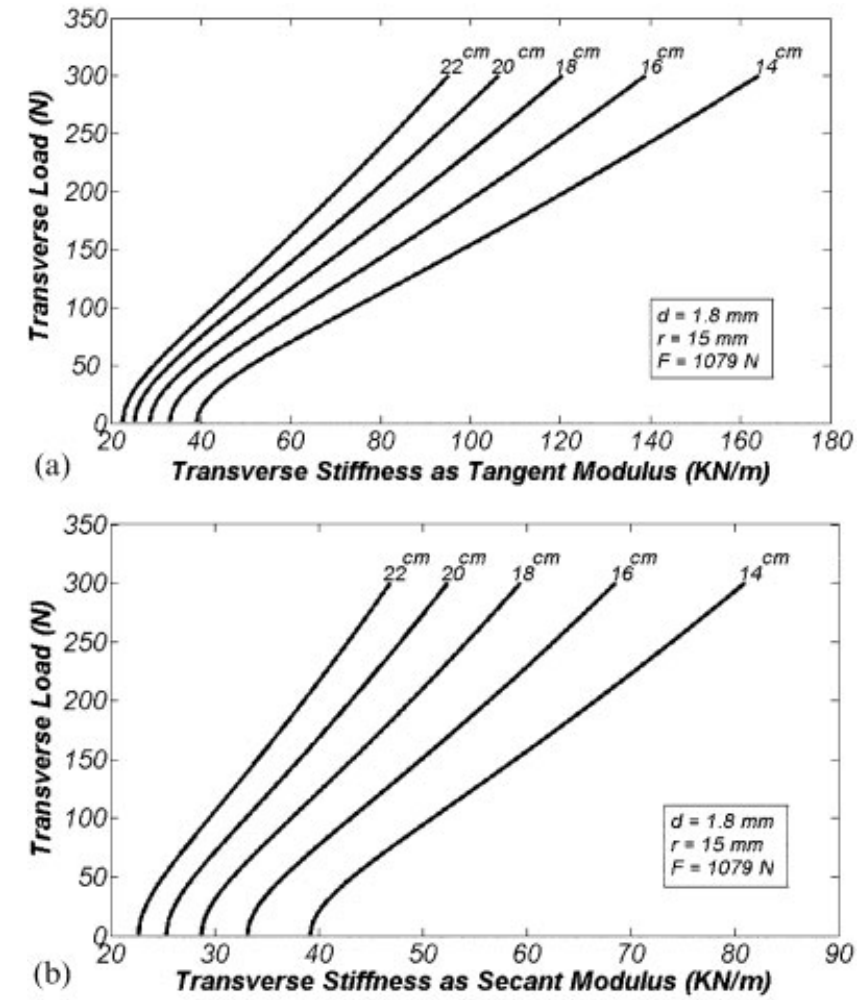

Fig. 6 Load versus stiffness curves for a wire under a given pre-tension with different wire spans, with stiffness defined (a) as the tangent modulus $\left(K_{\mathrm{t}}=P / y\right)$ (see equation (20)), and (b) as the secant modulus $\left(K_{\mathrm{s}}=P / y\right)$

the stiffness per $40 \mathrm{~mm}$ decrease in ring diameter predicted by the presented theoretical model varies from 40 per cent to 20 per cent. That is, it varies by \pm 10 per cent around a central value of 30 per cent.

\section{CONCLUSION}

The aim of this study was to provide a theoretical background to the behaviour of K-wires in Ilizarov ring fixators. A purely elastic tensile model was adopted. The tensile model assumption was based on published experimental $[\mathbf{1}]$, finite element $[\mathbf{1}$, 12], and theoretical studies $[5,12]$. Justification for purely elastic analysis was emphasized by the present findings (equation (8)). The resulting equations and their implication can be used to calculate final tension, reduction in tension due to slippage (or similar effects at the clamps), transverse deflection, angle of deflection, as well as stiffness of the K-wires under different parameters. Parametric discussion and implications of the equations elucidate and describe previously published data.

\section{REFERENCES}

1 Watson, M. A., Matthias, K. J., Maffulli, N., and Hukins, D. W. L. Yielding of the clamped-wire system in the Ilizarov external fixator. Proc. Instn Mech. Engrs, Part H: J. Engineering in Medicine, 2003, 217, 367-374.

2 Mullins, M. M., Davidson, A. W., Goodier, D., and Barry, M. The biomechanics of wire fixation in the Ilizarov system. Injury, Int. J. Care Injured, 2003, 34, 155-157.

3 Aronson, J. Current concepts review - limblengthening, skeletal reconstruction, and bone transport with the Ilizarov method. J. Bone Jt Surg. Am., 1997, 79, 1243-1258.

4 Aronson, J. and Harp Jr, J. H. Mechanical considerations in using tensioned wires in a transosseous external fixation system. Clin. Orthop. Related Res., 1992, 280, 23-29.

5 Nikonovas, A. and Harrison, A. J. L. A simple way to model the wires in Ilizarov wires used in ring fixators: analysis of the wire stiffness effect on overall fixator stiffness. Proc. IMechE, Part $H$ : J. Engineering in Medicine, 2005, 219, 31-42.

6 Donga, Y., Saleh, M., and Yang, L. Quantitative assessment of tension in wires of fine-wire external fixators. Med. Engng Physics, 2005, 27, 63-66.

7 Bronson, D. G., Samchukov, M. L., Birch, J. G., Browne, R. H., and Ashman, R. B. Stability of external circular fixation: a multi-variable biomechanical analysis. Clin. Biomechanics, 1998, 13, 441-448.

8 Fleming, B., Paley, D., Kristiansen, T., and Pope, M. A biomechanical analysis of the Ilizarov external fixator. Clin. Orthop. Related Res., 1989, 241, 95-105.

9 Watson, M. A., Mathias, K. J., and Maffulli, N. External ring fixators: an overview. Proc. Instn Mech. Engrs, Part H: J. Engineering in Medicine, 2000, 214, 459-470.

10 Wolf, S., Janousek, A., Pfeil, J., Veith, W., Haas, F., Duda, G., and Claes, L. The effect of external mechanical stimulation on the healing of diaphyseal osteotomies fixed by flexible external fixation. Clin. Biomechanics, 1998, 13, 359-364.

11 Zhang, G. Geometric and material nonlinearity in tensioned wires of an external fixator. Clin. Biomechanics, 2004, 19, 746-750.

12 Hillard, P. J., Harrison, A. J., and Atkins, R. M. The yielding of tensioned fine wires in the Ilizarov frame. Proc. Instn Mech. Engrs, Part H: J. Engineering in Medicine, 1998, 212, 37-47.

13 Zhang, G. Avoiding the material nonlinearity in an external fixation device. Clin. Biomechanics, 2004, 19, 513-518.

14 Watson, M. A., Mathias, K. J., Maffulli, N., and Hukins, D. W. L. The effect of clamping a tensioned wire: implications for the Ilizarov external fixation system. Proc. Instn Mech. Engrs, Part H: J. Engineering in Medicine, 2003, 217, 91-98.

15 Roark, R. J. and Young, W. C. Formulas for stress and strain, 1975 (McGraw-Hill, New York). 
16 Leonard, J. W. Tensioned structures, behaviour and analysis, 1998 (McGraw-Hill, New York).

17 Nash, W. A. Theory and problems of strength of materials, 2nd edition, 1977, pp. 15-16 (McGrawHill, New York).

18 Podolsky, A. and Chao, E. Y. S. Mechanical performance of Ilizarov circular external fixators in comparison with other external fixators. Clin. Orthop. Related Res., 1993, 293, 61-70.

\section{APPENDIX}

\section{Notation}

A

$c$

$d$

$D$

\section{E}

F

$H$

I

K

$K_{\mathrm{s}}$ cross-sectional area of the wire

constant independent of $L$, in $K=c / L$

wire diameter

internal diameter of the ring

total elongation induced in the wire

segment connecting the bone to the

clamp (due to both pre-tensioning and the lateral loading)

Young's modulus of elasticity

pre-tension

horizontal component of the reaction force at the support

second moment of area of the cross-

section of the K-wire

stiffnesses

stiffness defined as the secant

modulus
$K_{\mathrm{t}}$

$L$

$L^{\prime}$

$L_{-1}$

$L_{0}$

$p(y)$

$P$

$r$

$r_{\mathrm{g}}$

$T$

$y$

$\delta_{0}$

$\Delta L$ stiffness defined as the tangent modulus

total length of the K-wire

length of the wire segment connecting the bone to the clamp after both pre-tensioning and lateral loading length of the wire segment connecting the bone to the clamp before pretension (i.e. tension free)

length of the wire segment connecting the bone to the clamp after pretension

$=P$ (the lateral load), considered as a function of the displacement $y$

concentrated load applied transversely to the K-wire outer radius of the bone at the point of wire insertion

radius of gyration of the cross-

section $=\sqrt{I / A}$

final tension after both pretensioning and lateral loading maximum transverse deflection of the wire

angle of deflection

elongation induced in $L_{-1}$ due to pretension

elongation induced in $L_{0}$ due to lateral loading 